\title{
Study on Chemical Reactions of Ions and Neutral Particles on the Wall with DSMC Method
}

\author{
Yongyuan $\mathrm{Su}^{\mathrm{a}}$, Jie $\mathrm{Li}^{\mathrm{b}}$ and Zhenglei Fan ${ }^{\mathrm{c}, *}$ \\ National University of Defense Technology, Changsha 410073, China \\ a1156372010@qq.com, blijie_gfkd@163.com, ${ }^{\mathrm{a}} 2211302736 @ q q . c o m$
}

Keywords: DSMC method, dissociation-recombination reaction, chemical reactions on the wall.

\begin{abstract}
Plasma plumes exist widely in the electric propulsion systems. Neutral particles, ions, and metal particles are included in the flow field. Complex collisions occur between the particles. Particles will collide with the wall surface when they move towards the wall, then some particles will reflect back, the others will react on the wall with a certain probability. Chemical reactions on the wall have a significant effect on the distribution of the particles and lead to the change of density in the flow field. The chemical reaction models on the wall are studied based on the direct simulation of Monte Carlo method (DSMC). Five types of particles including molecular hydrogen $\mathrm{H}_{2}$, atomic hydrogen $\mathrm{H}$, atomic Titanium $\mathrm{Ti}$, ion $\mathrm{H}_{2}{ }^{+}$, and ion $\mathrm{H}^{+}$are studied in the flow field, there are chemical reactions for these particles with a certain probability on the wall. The numerical simulation results show that the number density of molecular hydrogen $\mathrm{H}_{2}$ increases obviously, the number density of atomic hydrogen $\mathrm{H}$ increases slightly, and the number density of ion $\mathrm{H}^{+}$decreases significantly after adding the chemical reaction models on the wall, indicating that the chemical reactions on the wall have a significant influence on the distribution of the particles in the flow field. The study in this paper provides basic data for further research on plasma plumes by the numerical simulation.
\end{abstract}

\section{Introduction}

Plasma plumes exist widely in aerospace propulsion [1], industrial coating [2,3] and nuclear reactors [4,5] such as plasma thrusters in the aerospace propulsion system [6] and micro-cathode vacuum arc thrusters for microsatellites [7]. In the thruster devices described above, plasma is injected to the environmental medium at an extremely high speed, and a feather-like jet flow called a plume is diffused into the surrounding environment rapidly. Plasma plume is often used in the high stability plasma devices, but the design of the devices has mostly relied on engineering experience, which is hinder the development of the performance. To better improve the performance of plasma devices, the transport characteristics of the plasma plumes need to be further studied.

The plasma plumes involve a series of complex dynamic behaviors in the transportation, and the multi-field coupling characteristics of the flow field and the electric field are obvious. The chemical reactions of the ions and neutral particles on the wall have been studied by DSMC method in this paper. The DSMC method [8] is widely used to solve the problem of rarefied fluid dynamics. Bird [9] proved the consistency of the DSMC method and the Boltzmann equation by mathematical derivation method. In view of the physical essence of DSMC, various numerical models are introduced to simulate complex physical and chemical processes [10], especially for the thermochemical non-equilibrium [11], radiation [12], and gas-solid interaction [13] and other complex problems.

\section{Chemical reaction models on the wall}

The chemical reaction is the process in which each particle collides with the wall surface and reacts with the metal on the wall to generate new particles. In the course of reaction, the charge of ions, the number of atoms, and the speed of the particles will change. At the same time, a small amount of particles do not leave the wall after reacting with the wall but finally are absorbed by the wall. Therefore, the adsorption of the particles belongs to the part of the chemical reactions on the wall, but the adsorption principle is complex and the types of the wall, the particles, the roughness of 
the wall surface need to be considered, and the adsorption on the wall surface has a small share of the chemical reactions. Therefore, the adsorption of particles on the wall surface can be neglected, and only other chemical reactions on the wall surface will be considered. At the same time, in the chemical reactions on the wall, the reactions involved metal atomic on the wall occur very little, and therefore the chemical reactions involved by the particles of metal atomic not considered. Although electrons participate in the chemical reactions, but electrons move faster by several orders of magnitude then ions. Considering the electron participation in the chemical reactions will greatly increase the amount of calculation. Therefore, the chemical reactions on the wall refer only to the chemical reactions of the ions and neutral particles on the wall surface, and the particles related to the wall are not considered in this paper.

During the process of ions movement, the types of the boundary that particles pass through need to be determined. When particles move to the wall, it is labeled whether a chemical reaction occurs according to the probability of chemical reaction. When chemical reactions occur, the particle are labeled, the types of chemical reactions and the partner molecular of the labeled molecular must be marked too. When all the simulated molecules have finished movement, all the molecules are checked individually. As for the labeled molecules, according to the types of chemical reactions, new particles are produced, and energy distributions are disposed.

The dissociation reaction of polyatomic ions and the recombination reaction of monoatomic are included in the chemical reactions model on the wall. For the dissociation reaction of polyatomic ions, the probability is determined by the energy distribution. Reactions can occur only when the vibrational energy is more than the dissociation energy. The first step in judging whether or not the dissociation reaction occurs is to determine whether the vibration energy participates in the energy distribution. In the DSMC algorithm, the reciprocal of the number of vibration energy relaxation collisions is greater than a random number. Generally, the number of vibration energy relaxation collisions is taken as 50 [14]. If the vibrational energy is involved in the energy distribution, the total energy of the molecule is calculated, including relative translational energy, rotational energy, and vibrational energy. The second step in judging whether or not the dissociation reaction occurs is to determine whether the total energy of the molecule is greater than the dissociation energy. If the total energy of the molecule is greater than the dissociation energy, the energy level after the molecular excitation is calculated according to the acceptance rejection samplingmethod. The final step is to determine whether the energy level of the molecule is greater than the energy level required for dissociation. If the energy level of the molecule is greater than the energy level required for dissociation, the dissociation reaction is considered to occur, and the molecule is marked. After determining that the molecule has undergone a dissociation reaction, the molecular information after the reaction is calculated. The total number of molecules in the flow field is increased by one, and the position and the velocity of the two molecules are the same, and the position of two molecules accord with the position of the molecule before dissociation, and then the rotation energy is converted into the relative translational energy of the two molecules.

For the recombination reaction of single atom, the probability of the reaction was calculated based on Bird's phenomenological chemical reaction model [8]. Bird studied the relationship between the macroscopic reaction rate constant and the microscopic chemical reaction probability:

$$
\frac{\sigma_{R}}{\sigma_{T}}=\frac{\pi^{1 / 2} \varepsilon \Lambda T_{r e f}^{\eta}}{2 \sigma_{r e f}\left(k T_{r e f}\right)^{\eta-1+\omega_{A B}}} \frac{\Gamma\left(\bar{\xi}+5 / 2-\omega_{A B}\right)}{\Gamma(\bar{\xi}+\eta+3 / 2)}\left(\frac{m_{r}}{2 k T_{r e f}}\right)^{1 / 2} \frac{\left(E_{c}-E_{a}\right)^{\eta+\bar{\xi}+1 / 2}}{E_{c}^{\bar{\xi}+3 / 2-\omega_{A B}}}
$$

Usually the chemical reaction probability is expressed by $\sigma_{\mathrm{R}} / \sigma_{\mathrm{T}} ; \sigma_{\mathrm{R}}$ is reaction cross-sections; $\sigma_{\mathrm{T}}$ is total collision cross-section; $\boldsymbol{\varepsilon}$ is the symmetry factor, 2 means the type of particles are the same, and 1 are different; $\Lambda$ and $\eta$ is the constant related to the chemical rate; $T_{\text {ref }}$ is the reference temperature; $\sigma_{\text {ref }}$ is the reference collision cross-sectional area; $\mathrm{k}$ is the Boltzmann constant; $\bar{\xi}$ is the mean internal freedom of the collision pair; $\omega_{\mathrm{AB}}$ is the viscosity-temperature index of the collision pair; $\mathrm{m}_{\mathrm{r}}$ is the degeneracy mass; $E_{c}$ is the total energy of the collision pair, including internal energy and relative 
translational energy, the rotational energy, vibration energy and electron energy are include in internal energy; $E_{a}$ is the activation energy of the reaction.

According to the probability, it is judged whether or not a recombination reaction occurs, and the molecule is marked. After confirming that the reaction has occurred, the information of the product needs to be calculated. The speed of produced molecule is the relative centroidal velocity of the two molecules before reaction, and the position is the same as where any one of the molecules is before reaction. Assign the information of the last molecule to the other molecule of the recombination reaction and subtract 1 from the number of total molecules. Then a molecule is randomly selected in the same grid, and the total energy is distributed between the produced molecule and the selected molecule. The total energy distributed by the recombination reaction includes the relative translational energy of the molecules that undergo the chemical reaction, the relative translational energy of the produced molecule and the selected molecule, and the dissociation energy of the produced molecule. The first step is to determine the maximum vibration energy level that the total energy can reach. Then the vibration energy is calculated by the acceptance-rejection sampling method. The rotational energy is then redistributed. The relative translational energy of the produced molecular and the selected molecular is the total energy minus the vibrational energy and the rotational energy. Finally, the relative translational energy is assigned to the produced molecule and the selected molecule.

\section{Numerical simulation and results analysis}

\subsection{Geometric model and calculation conditions}

The geometric model in this paper is shown in the following figure:

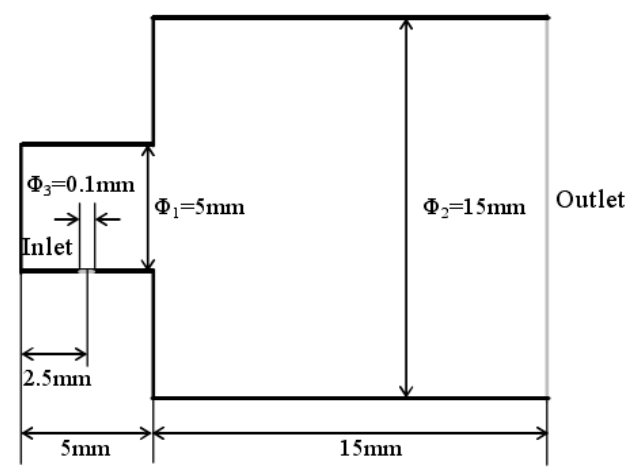

Fig. 1 Geometric model

The molecular hydrogen $\mathrm{H}_{2}$, atomic hydrogen $\mathrm{H}$, atomic Titanium $\mathrm{Ti}$, ion $\mathrm{H}_{2}{ }^{+}$, and ion $\mathrm{H}^{+}$are injected to the vacuum cylinder, and the proportions of each particles are: $15 \%, 15 \%, 40 \%, 15 \%$, and $15 \%$. The temperature of particles is $3000 \mathrm{~K}$, and the initial velocity of particles is $5000 \mathrm{~m} / \mathrm{s}$. The total density of injected particles changes as follows:

$$
f=\left\{\begin{array}{cc}
5 \times 10^{24} \times \sin (\pi-b \cdot(a-t)) & 0 \leq t \leq a \\
0 & a \leq t
\end{array}\right.
$$

Where $\mathrm{a}=5 \mathrm{E} 10-6, \mathrm{~b}=5.46 \mathrm{E} 5$.

The injection surface is a semicircular surface with a diameter of $0.1 \mathrm{~mm}$ on the side wall of the small cylinder, and the particle velocity accords with the equilibrium distribution based on the macroscopic velocity. The bottom and side surface of the small cylinder and the side surface of the large cylinder are the wall boundary conditions. Regardless the material type of wall, the wall temperature is $300 \mathrm{~K}$. The molecular collides with the wall according to a diffuse model or reaction with wall when the molecules move to the wall. The right side of the large cylinder is the exit boundary condition. When the molecule moves to the exit boundary, the information of molecule will be deleted directly. The axis of two cylinders is symmetric boundary condition. When a molecule 
enters the other side through the plane of symmetry, there will be one molecule moving in from the other side at the same tangential velocity and the reverse normal velocity.

The Bird's No-Time Counting model (NTC), the Larsen-Borgnakke energy distribution model, the Variable-Hard-Sphere model (VHS) of molecular, and the completely diffuse reflection model on the wall are used in this paper, the influence of magnetic field is ignored.

\subsection{The simulation of the chemical reactions on the wall and the results analysis}

The dissociation of the molecular hydrogen $\mathrm{H}_{2}$ and the recombination of the atomic hydrogen $\mathrm{H}$ are considered in the flow field in this paper. The chemical reactions considered on the wall are shown in the following Table1. The probability is obtained from the reference [15].

Table 1 The types and the probability of the chemical reactions on the wall

\begin{tabular}{cccc}
\hline $\begin{array}{c}\text { Types of the } \\
\text { chemical reactions }\end{array}$ & $\begin{array}{c}\text { Probability of the } \\
\text { chemical reactions }\end{array}$ & $\begin{array}{c}\text { Types of the } \\
\text { chemical reactions }\end{array}$ & $\begin{array}{c}\text { Probability of the } \\
\text { chemical reactions }\end{array}$ \\
\hline $\mathrm{H}^{+}+$Wall $\rightarrow \mathrm{H}_{2}$ & 0.4 & $\mathrm{H}_{2}{ }^{+}+$Wall $\rightarrow \mathrm{H}$ & 0.8 \\
$\mathrm{H}^{+}+$Wall $\rightarrow \mathrm{H}$ & 0.6 & $\mathrm{H}+\mathrm{Wall} \rightarrow \mathrm{H}_{2}$ & 0.4 \\
$\mathrm{H}_{2}{ }^{+}+\mathrm{Wall} \rightarrow \mathrm{H}_{2}$ & 0.2 & $\mathrm{H}+\mathrm{Wall} \rightarrow \mathrm{H}$ & 0.6 \\
\hline
\end{tabular}

The distribution of the molecular hydrogen $\mathrm{H}_{2}$, atomic $\mathrm{H}$, and ion $\mathrm{H}^{+}$are affected obviously by chemical reaction on the wall. Fig. 2 shows the number density of molecular $\mathrm{H}_{2}$ along the $\mathrm{Y}$ direction at the symmetry plane $\mathrm{X}=0.001 \mathrm{~m}$ over time. It can be seen from the figure that the number density of molecular $\mathrm{H}_{2}$ increases significantly after the chemical reaction is added to the wall, because both the ion $\mathrm{H}^{+}$and the atomic $\mathrm{H}$ which move to the wall will generate $\mathrm{H}_{2}$ with a certain probability in the flow field, and the numbers of the ion $\mathrm{H}^{+}$and the atomic $\mathrm{H}$ are large, so the number density of molecular $\mathrm{H}_{2}$ increases significantly. In addition, as it can be seen from the figure, there is no difference in the number density of the molecular $\mathrm{H}_{2}$ at the same position along $\mathrm{Y}$ direction when the time is $1 \mathrm{E}-6 \mathrm{~s}$, whether or not the chemical reactions exist on the wall. As the time increases, the number of the molecular $\mathrm{H}_{2}$ increases in the same position along $\mathrm{Y}$ direction. The number density of molecular $\mathrm{H}_{2}$ increases after adding the chemical reaction on the wall by almost one order of magnitude compared to that without reaction on the wall when the time is 7E-6s, indicating that the influence of the number density of molecular $\mathrm{H}_{2}$ is increasing by the chemical reaction on the wall over time. It can also be seen from the figure that the effect of the chemical reaction on the number density of the molecular $\mathrm{H}_{2}$ gradually increases with the increase of the $\mathrm{Y}$ distance when the time is $1 \mathrm{E}-6 \mathrm{~s}$, and the effect is the greatest when the distance along $\mathrm{Y}$ direction is the largest, and at the other three different times, the influence of the reaction on the number density of the molecular $\mathrm{H}_{2}$ gradually decreases with the increase of $\mathrm{Y}$ distance. This is because that at the time is $1 \mathrm{E}-6 \mathrm{~s}$, the particle diffusion is not sufficient, and the reaction far away from the outlet of the nozzle has a major influence. After the time is $1 \mathrm{E}-6 \mathrm{~s}$, the particles diffuse sufficiently and all the wall of the small cylinder has a great influence on the flow distribution. At this time, the particles near the exit area of the nozzle are affected by a series of reactions on the wall, so the reaction on the wall in this area has a significant influence on the number density of the molecular $\mathrm{H}_{2}$.

To illustrate this problem, Fig. 3 shows a streamline when the time is $3 \mathrm{E}-6 \mathrm{~s}$. It can be seen from the figure that the particles collide with the wall surface when they move far away from the outlet wall of the nozzle, returning to the flow field and forming a three-dimensional vortex structure on the left side of the jet stream. When the particles move to the $\mathrm{X}=0.001 \mathrm{~m}$ position, they have experienced a series of reactions on the wall, so after the particles have diffused fully, the reactions on the wall have the greatest influence on the number density of the molecular $\mathrm{H}_{2}$ near the outlet of the nozzle.

Fig. 4 shows the number density of the atomic $\mathrm{H}$ along the $\mathrm{Y}$ direction on symmetry plane $\mathrm{X}=$ $0.001 \mathrm{~m}$ at different times. It can be seen from the figure that after the chemical reaction model is added, the number density of the atomic $\mathrm{H}$ increases, but the increase is not very obvious, because in the chemical reactions on the wall, the atomic $\mathrm{H}$ generates the molecular $\mathrm{H}_{2}$ with $40 \%$ probability, while the ion $\mathrm{H}_{2}{ }^{+}$generates $\mathrm{H}$ with the probability of $80 \%$, and the wall chemical reaction only occurs when the particles move to the wall, so the number density of the atomic $\mathrm{H}$ will increase, but the increasing trend is not obvious. It can be seen from the figure that the number density of the atomic $\mathrm{H}$ 
is reduced by the reaction on the wall near the inlet of the nozzle when the time is $1 \mathrm{E}-6 \mathrm{~s}$. This is because the particles have not yet diffused fully, and the diffusion rate of the particle of the atomic $\mathrm{H}$ is higher than that of the ion $\mathrm{H}_{2}{ }^{+}$. As a result, the probability of the reactions of the atomic $\mathrm{H}$ is larger than that of the ion of $\mathrm{H}_{2}{ }^{+}$on the wall, so the number density of the atomic $\mathrm{H}$ will decrease slightly. At other times, the particle diffusion is sufficient and the chemical reactions on the wall will be obvious.

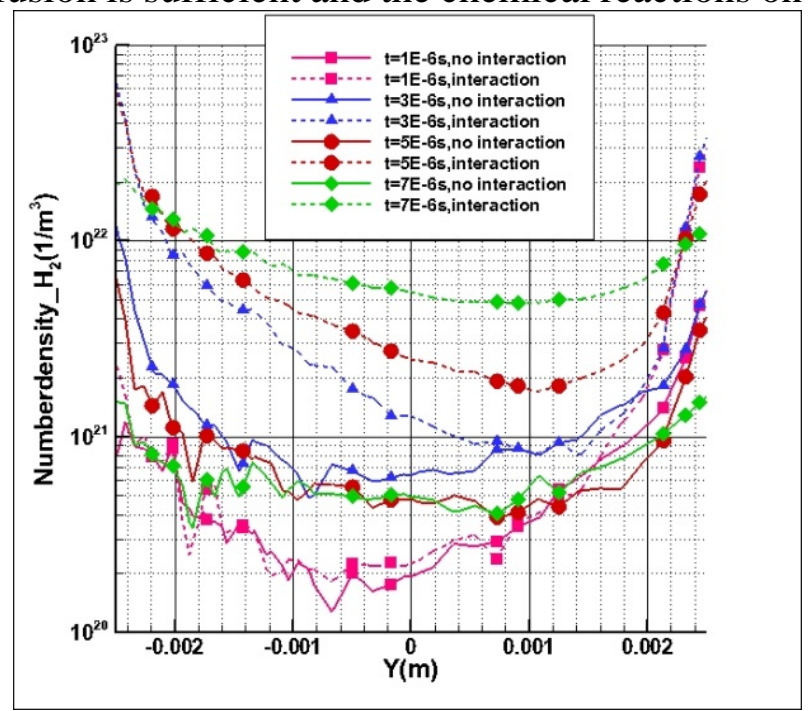

Fig. 2 The number density of the molecular hydrogen $\mathrm{H}_{2}$ along the

$\mathrm{Y}$ direction on symmetry plane $\mathrm{X}=0.001 \mathrm{~m}$ at different times

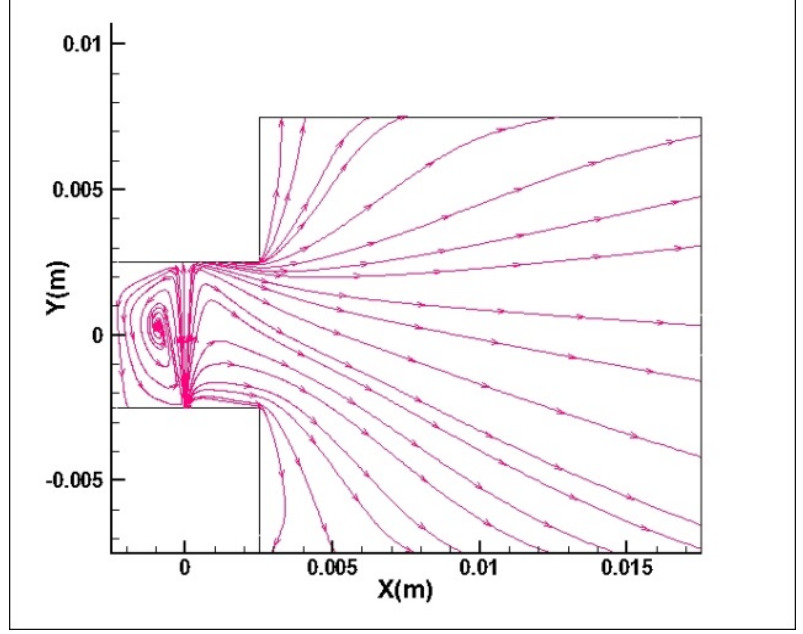

Fig. 3 The streamlines at the time 3E-6s

Fig. 5 shows the number density of the ion $\mathrm{H}^{+}$along the $\mathrm{Y}$ direction on symmetry plane $\mathrm{X}=$ $0.001 \mathrm{~m}$ at different times. It can be seen from the figure that the number density of the ion $\mathrm{H}^{+}$ decreases significantly after the reaction on the wall model is added. Because the ion $\mathrm{H}+$ generates $\mathrm{H}_{2}$ with the reaction probability of $40 \%$ on the wall, so the number density of the ion $\mathrm{H}^{+}$will be reduced obviously. It can be seen that the number density of the ion $\mathrm{H}^{+}$is lower than that of the atomic $\mathrm{H}$ with the same probability compared with Fig.3. Because the number density of ion $\mathrm{H}^{+}$is one order of magnitude lower than that of atomic $\mathrm{H}$, resulting in the number density change of the ion $\mathrm{H}^{+}$is more obvious.

Above all, it can be seen that the number density of the molecular hydrogen $\mathrm{H}_{2}$ increases significantly after the chemical reaction models are added, the number density of the ion $\mathrm{H}^{+}$decreases significantly, and the number density of the atomic hydrogen $\mathrm{H}$ increases slightly. It shows that the reaction on the wall has a great influence on the number density of the molecular $\mathrm{H}_{2}$ and the ion $\mathrm{H}^{+}$, and has little effect on the number density of the atomic $H$. The chemical reaction on the wall near the outlet of the nozzle is the most obvious, and the influence of number density change of molecular $\mathrm{H}_{2}$ and ion $\mathrm{H}^{+}$by the chemical reaction on the wall is more obvious with the increase of time. 


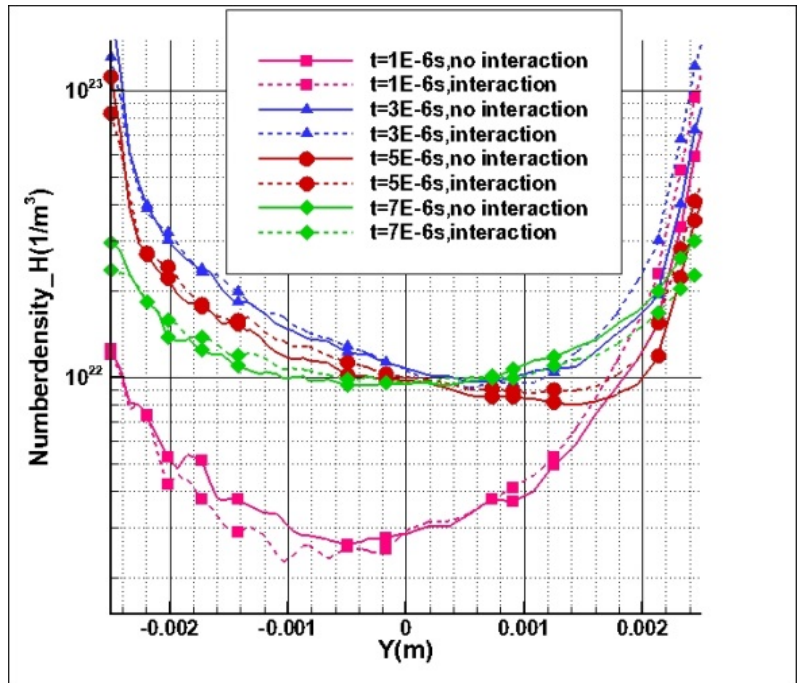

Fig. 4 The number density of the atomic $\mathrm{H}$ along the $\mathrm{Y}$ direction on the symmetry plane $\mathrm{X}=0.001 \mathrm{~m}$ at different times

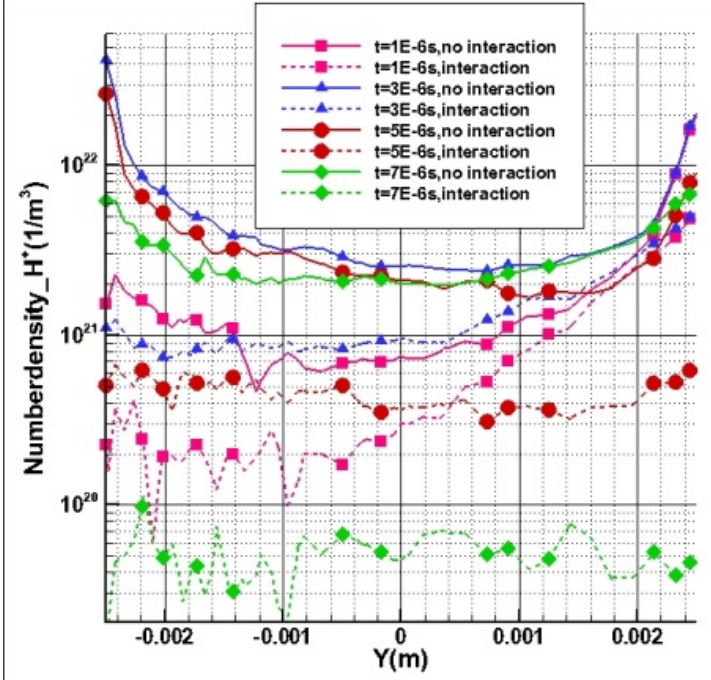

Fig. 5 The number density of the ion $\mathrm{H}^{+}$along the $\mathrm{Y}$ direction on symmetry plane $\mathrm{X}=0.001 \mathrm{~m}$ at different times

\section{Conclusion}

Some chemical reactions on the wall have been studied based on the DSMC method in this paper. The chemical reaction models on the wall has a great influence on the number density of the molecular hydrogen $\mathrm{H}_{2}$ and the ion $\mathrm{H}^{+}$, and has little effect on the number density of the atomic hydrogen $\mathrm{H}$. The number density change of particles near the outlet of the nozzle is the most obvious, and as the time increases, the number density change of the molecular hydrogen $\mathrm{H}_{2}$ and the ion $\mathrm{H}^{+}$ becomes more obvious due to the chemical reactions on the wall. This paper provides basic data for further research on plasma plumes, but the electric field, the electrons, and the magnetic field are neglected in this model, so the model still needs to be improved.

\section{Acknowledgments}

This paper is supported by NSAF, Grant No.U1730247.

\section{References}

[1] Smith B D, Boyd I D, Kamhawi H, et al. 2013.Hybrid-PIC modeling of a high-voltage, high-specific-impulse Hall thruster. 49th AIAA/ASME/SAE/ASEE Joint Propulsion Conference, 
San Jose, CA.

[2] Mozetič M, Ostrikov K, Ruzic D N, et al. 2014. Recent advances in vacuum sciences and applications.Journal of Physics D Applied Physics, Vol. 47(15).

[3] Hirata Y, Kato T, Choi J. 2015. DLC coating on a trench-shaped target by bipolar PBII. International Journal of Refractory Metals \& Hard Materials, Vol. 49(1).

[4] Francesco, Taccogna, Pierpaolo, et al. 2012. Kinetic divertor modeling. Chemical Physics, Vol. 398(1).

[5] Gleason-González C, Varoutis S, Hauer V, et al. 2014. Simulation of neutral gas flow in a tokamak divertorusing the Direct Simulation Monte Carlo method. Fusion Engineering \& Design, Vol. 89(7-8).

[6] Korkut B, Li Z, Levin D A. 2015. 3-D Simulation of Ion Thruster Plumes Using Octree Adaptive Mesh Refinement. IEEE Transactions on Plasma Science, Vol. 43(5).

[7] Brieda L, Zhuang T S, Keidar M. 2013. Near Plume Modeling of a Micro Cathode Arc Thruster. 49thAIAA/ASME/SAE/ASEE Joint Propulsion Conference, San Jose, CA.

[8] Bird G A. 2003. Molecular gas dynamics and the direct simulation of gas flows. Oxford: Clarendon Press.

[9] Bird G A. 1970. Direct simulation of the Boltzmann equation. Phys. Fluids, Vol. 13(13).

[10]Moss J, Price J, Dogra V, Hash D. 1995. Comparision of DSMC and experimental results for hypersonic external flow. AIAA Paper.

[11]Boyd I D. 1990. Rotational and vibrational nonequilibrium effects in rarefied hypersonic flows. Journal of Thermophysics and Heat Transfer, Vol. 4(4).

[12]Everson J, Nelson H F. 1993. Development and Application of a reverse Montel Carlo radiative transfer code for rocket plume base heating. AIAA Paper.

[13]Plastinin Y, Anfimov N, Baula G, Karabadzhk G, Khmelinin B. 1993. Modeling of aluminum oxide particle radiation in a solid propellant motor exhaust. AIAA paper.

[14] SHEN Qin. 2003. Rarefied gas dynamics. Beijin: National Defense Industry Press, No. 5.

[15]Boeuf J P, Hagelaar G J M, Sarrailh P, Fubiani G, Kohen N. 2011. Model of an inductively coupled negative Ion Source: II. Application to an ITER type source. Plasma Sources Sci. Technol, Vol. 20(1). 